\title{
A NOTE ON COBORDISM OF SURFACE LINKS IN $S^{4}$
}

\author{
MASAHICO SAITO \\ (Communicated by Frederick R. Cohen)
}

\begin{abstract}
Sato's idea of the asymmetric linking number is used in cyclic branched coverings to give an invariant of the cobordism of embedded surfaces in the 4-sphere.
\end{abstract}

In this article we consider the link cobordism of surface links in $S^{4}$. We work in the smooth category.

Let $L=J \cup K$ be a link in $S^{4}$, where $J, K$ are embedded, oriented connected surfaces. $L$ is called semi-boundary $[\mathrm{S}]$ if each component bounds an embedded, orientable 3-manifold in $S^{4}$ which misses the other component. Sato [S] defined the asymmetric linking number, denoted by alk $(J, K)$, to be the nonnegative generator of the image of $H_{1}(K: \mathbf{Z}) \rightarrow H_{1}\left(S^{4} \backslash J: \mathbf{Z}\right) \cong \mathbf{Z}$. He proved that a link is semi-boundary iff

$$
\operatorname{alk}(J, K)=0=\operatorname{alk}(K, J),
$$

and being semi-boundary is preserved under link cobordism. We call two surface links $L_{0}=J_{0} \cup K_{0}$ and $L_{1}=J_{1} \cup K_{1}$ cobordant if there are disjointly embedded, orientable 3-manifolds $C$ and $E$ in $S^{4} \times I$ such that $\partial C=J_{0} \cup\left(-J_{1}\right)$, $\partial E=K_{0} \cup\left(-K_{1}\right)$, and $C, E$ are homeomorphic to $J_{0} \times I, K_{0} \times I$, respectively, where we regard $L_{i}$ as lying in $S^{4} \times\{i\}$. A link is called null-cobordant if it is cobordant to the standardly embedded surfaces (which bound disjoint handlebodies) in $S^{4}$. Thus alk can be regarded as the first obstruction to links being null-coborciant, and we focus on semi-boundary links from now on. The Sato-Levine invariant was defined [S] for semi-boundary links, and Cochran [C] defined the derived series of this invariant. In this paper we observe that the covering asymmetric linking number can be used as a link cobordism invariant and give examples of links with vanishing Sato-Levine invariant and trivial derivatives which belong to different cobordism classes.

Let $L=J \cup K$ be a 2-component, oriented semi-boundary link. Consider the $n$-fold cyclic branched covering $M$ of $S^{4}$ along $J$, where $n=p^{r}$ is a prime power. Then there are $n$ lifts $k_{0}, \ldots, k_{n-1}$ of $K$ to $M$ since $L$ is

Received by the editors December 5, 1989.

1980 Mathematics Subject Classification (1985 Revision). Primary 57Q45; Secondary 57Q60.

Key words and phrases. Surface links, cobordism, covering asymmetric linking number. 
semi-boundary. We can assume that $k_{j+1}=\tau k_{j}$ where $\tau$ is the generator of covering translations. We regard $n$ as 0 and $n+1$ as 1 , so that the indices of lifts are regarded as lying in $\mathbf{Z}_{n}$.

Lemma 1. $H_{1}\left(M \backslash k_{j}: \mathbf{Q}\right)$ is isomorphic to either 0 or $\mathbf{Q}$. Furthermore, this is a link cobordism invariant. More precisely, let $L_{i}=J_{i} \cup K_{i}(i=0,1)$ be cobordant links and $M_{i}, k_{j}^{i}$ be their cyclic branched coverings and the lifts of $K_{i}$ respectively $(i=0,1, j=0, \ldots, n-1)$. Then

$$
H_{1}\left(M_{0} \backslash k_{j}^{0}: \mathbf{Q}\right) \cong H_{1}\left(M_{1} \backslash k_{j}^{1}: \mathbf{Q}\right) .
$$

In particular, this is $\mathbf{Q}$ if $L$ is null-cobordant.

The proof is given later. If $H_{1}\left(M \backslash k_{j}: \mathbf{Q}\right)=0$, then $L$ is not null-cobordant. Thus we focus on links with $H_{1}\left(M \backslash k_{j}: \mathbf{Q}\right)=\mathbf{Q}$. Consider the following homomorphism

$$
H_{1}\left(k_{j}: \mathbf{Q}\right) \rightarrow H_{1}\left(M \backslash k_{0}: \mathbf{Q}\right) \cong \mathbf{Q} .
$$

Definition 2. Define $\xi_{j}^{n}=0$ if this homomorphism is zero, $\xi_{j}^{n}=1$ otherwise $(j=1, \ldots, n-1)$.

Theorem 3. $\xi_{j}^{n} \in \mathbf{Z}_{2}, j \in \mathbf{Z}_{n} \backslash\{0\}$, are link cobordism invariants.

Proof of Lemma 1. Let $X_{n}$ be the $n$-fold cyclic (unbranched) covering ( $X_{\infty}$ denotes the infinite cyclic covering) of $X=\overline{S^{4} \backslash N(J)}$. Then we have an exact sequence ([S-S]) with integral coefficient

$$
\cdots \rightarrow H_{q}\left(X_{\infty}\right) \stackrel{t^{n}-1}{\longrightarrow} H_{q}\left(X_{\infty}\right) \rightarrow H_{q}\left(X_{n}\right) \rightarrow H_{q-1}\left(X_{\infty}\right) \rightarrow \cdots,
$$

where $t$ is the homomorphism induced from the generator of covering transformations. Thus we have

$$
\begin{array}{ccccc}
H_{1}(X) & \rightarrow & H_{0}\left(X_{\infty}\right) & \stackrel{t-1}{\longrightarrow} & \|_{0} \\
\| & H_{0}\left(X_{\infty}\right)
\end{array} .
$$

Hence $(t-1): H_{1}\left(X_{\infty}: \mathbf{Z}\right) \rightarrow H_{1}\left(X_{\infty}: \mathbf{Z}\right)$ is surjective. Therefore $\left(t^{n}-1\right)=$ $(t-1)^{n}: H_{1}\left(X_{\infty}: \mathbf{Z}_{p}\right) \rightarrow H_{1}\left(X_{\infty}: \mathbf{Z}_{p}\right)$ is also surjective $\left(n=p^{r}\right.$ is a prime power). Again using the exact sequence, we have $H_{1}\left(X_{n}: \mathbf{Z}_{p}\right)=\mathbf{Z}_{p}$. But the lift of the meridian of $J$ represents a nontrivial element of infinite order in $H_{1}\left(X_{n}: \mathbf{Z}\right)$. Hence $H_{1}\left(X_{n}: \mathbf{Q}\right)=\mathbf{Q}$. Thus we have $H_{1}(M: \mathbf{Q})=0$. A Mayer-Vietoris sequence with $\mathbf{Q}$-coefficients gives

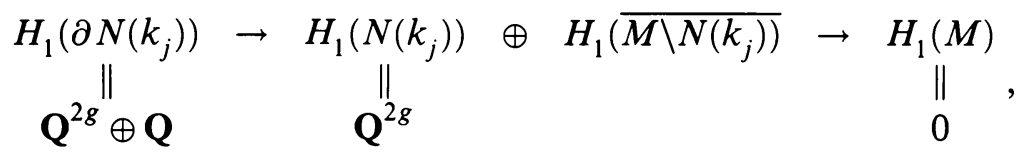

where $g$ is the genus of $K$. Hence $H_{1}\left(M \backslash k_{j}: \mathbf{Q}\right)=0$ or $\mathbf{Q}$. 
Let $L_{i}=J_{i} \cup K_{i}(i=0,1)$ be cobordant links via $C, E$, and let $W$ be the $n$-fold cyclic branched covering of $S^{4} \times I$ along $C, E_{j} \quad(j=0, \ldots$, $n-1)$ be the lifts of $E$ to $W$. Then $\partial E_{j}=k_{j}^{0} \cup\left(-k_{j}^{1}\right)$, where $k_{j}^{i}$ are the lifts of $K_{i}$ to $M_{i}$, the $n$-fold branched covering of $S^{3}$ along $J_{i}$. (Note that we have exactly $n$ lifts of $E$ because $L_{i}$ 's are semi-boundary and $E$ is homeomorphic to the product $K_{0} \times I$.) The same argument shows that $H_{1}(W: \mathbf{Q})=0$ and $H_{1}\left(W \backslash E_{0}: \mathbf{Q}\right)=0$ or $\mathbf{Q}$.

We need to know the homomorphism $H_{2}\left(M_{i}\right) \rightarrow H_{2}(W)$. Let $Y=$ $\overline{S^{4} \times I \backslash N(C)}, Y_{n}$ (resp. $Y_{\infty}$ ) be the $n$-fold (resp. infinite) cyclic covering of $Y$. Let $X^{i}=\frac{S^{4} \backslash J_{i}}{}, X_{n}^{i}$ (resp. $X_{\infty}^{i}$ ) be the $n$-fold (resp. infinite) cyclic covering of $X^{i}$. Since $(t-1): H_{1}\left(X_{\infty}^{i}: \mathbf{Z}\right) \rightarrow H_{1}\left(X_{\infty}^{i}: \mathbf{Z}\right)$ is surjective, it is an isomorphism (because $H_{1}\left(X_{\infty}^{i}: \mathbf{Z}\right)$ is a finitely generated module over a Noetherian ring $\Lambda=\mathrm{Z}\left[t, t^{-1}\right]$, see [S-S]). Also we have $H_{2}\left(Y, X^{i}\right)=0$ since the inclusion induces an isomorphism $H_{*}\left(X^{i}\right) \cong H_{*}(Y)$. Therefore we have the following commutative diagram with $\mathbf{Z}$-coefficients:

$$
\begin{aligned}
& 0 \rightarrow \begin{array}{ccccc}
H_{2}\left(X_{\infty}^{i}\right) \\
\downarrow
\end{array} \quad \stackrel{t-1}{\longrightarrow} \quad \begin{array}{c}
H_{2}\left(X_{\infty}^{i}\right) \\
\downarrow
\end{array} \quad \rightarrow \quad \begin{array}{c}
H_{2}\left(X^{i}\right) \\
\downarrow
\end{array} \quad \rightarrow \quad 0 \\
& 0 \rightarrow \begin{array}{ccccc}
H_{2}\left(Y_{\infty}\right) & \stackrel{t-1}{\longrightarrow} & H_{2}\left(Y_{\infty}\right) & \rightarrow & H_{2}(Y) \\
\downarrow & \rightarrow & 0
\end{array} \\
& 0 \rightarrow H_{2}\left(Y_{\infty}, X_{\infty}^{i}\right) \stackrel{t-1}{\longrightarrow} H_{2}\left(Y_{\infty}, X_{\infty}^{i}\right) \rightarrow 0
\end{aligned}
$$

Note that $i_{*}: H_{2}\left(X^{i}\right) \rightarrow H_{2}(Y)$ (the homomorphism induced from the inclusion map) is an isomorphism, and $H_{2}\left(X^{i}\right) \cong \mathbf{Z}^{2 h}$, where $h$ is the genus of $J_{i}$.

Consider the following splitting

$$
H_{2}\left(X_{\infty}^{i}: \mathbf{Q}\right) \cong F\left(X_{\infty}^{i}: \mathbf{Q}\right) \oplus T\left(X_{\infty}^{i}: \mathbf{Q}\right)
$$

where $F(), T()$ denote the free and torsion part of $H_{2}()$ as a $\Gamma=\Lambda \otimes_{\mathbf{Z}} \mathbf{Q}$ module respectively ( $\Gamma$ is a PID). Furthermore, $T\left(X_{\infty}^{i}: \mathbf{Q}\right)=T^{0}\left(X_{\infty}^{i}: \mathbf{Q}\right) \oplus$ $T^{1}\left(X_{\infty}^{i}: \mathbf{Q}\right)$, where

$$
T^{0}\left(X_{\infty}^{i}: \mathbf{Q}\right) \cong \Gamma /(t-1)^{p_{1}} \oplus \cdots \oplus \Gamma /(t-1)^{p_{e}}
$$

is the $(t-1)$-summand and $T^{1}()$ is the $(t-1)$-free summand.

Comparing the above sequence to the following sequence

$$
0 \rightarrow \Gamma /(t-1) \rightarrow \Gamma /(t-1)^{p_{k}} \stackrel{t-1}{\longrightarrow} \Gamma /(t-1)^{p_{k}} \rightarrow \Gamma /(t-1) \rightarrow 0,
$$

where $\Gamma /(t-1) \cong \mathbf{Q}$, we conclude that $T^{0}\left(X_{\infty}^{i}\right)=0$. (The same is true for $H_{2}\left(Y_{\infty}\right)$ and $H_{2}\left(Y_{\infty}, X_{\infty}^{i}\right)$.)

Hence $T\left(X_{\infty}^{i}: \mathbf{Q}\right) \cong \Gamma / \lambda_{1} \oplus \cdots \oplus \Gamma / \lambda_{m}$ where $\lambda_{j}$ is normalized so that $\lambda_{j} \in \Lambda$ and coefficients are relatively prime $(j=1, \ldots, m)$. 
On the other hand, $\operatorname{Cok}\left(t-1: H_{2}\left(X_{\infty}^{i}: \mathbf{Z}\right) \rightarrow H_{2}\left(X_{\infty}^{i}: \mathbf{Z}\right)\right)$ is isomorphic to $H_{2}\left(X_{\infty}^{i}\right) \otimes_{\Lambda} \mathbf{Z}$, where $\mathbf{Z}$ is regarded as $\Lambda$-module via the augmentation map $\Lambda \rightarrow \mathbf{Z}, t \rightarrow 1$ [S-S]. Since $H_{2}(X)$ is torsion free, we have $\lambda_{j}(1)= \pm 1, j=$ $1, \ldots, m$ [S-S]. Then the same argument as Theorem 3 in [Sum] shows that

$$
\operatorname{Cok}\left(t^{n}-1: T\left(X_{\infty}^{i}: \mathbf{Q}\right) \rightarrow T\left(X_{\infty}^{i}: \mathbf{Q}\right)\right)=0 \quad\left(n=p^{r}\right) .
$$

The same is true for $H_{2}\left(Y_{\infty}, X_{\infty}^{i}\right)$ and we have $H_{2}\left(Y_{n}, X_{n}^{i}: \mathbf{Q}\right)=0$ (since $H_{2}\left(Y_{\infty}, X_{\infty}^{i}\right)$ is $\Gamma$-torsion $)$, and hence $i_{*}: H_{2}\left(X_{n}^{i}: \mathbf{Q}\right) \rightarrow H_{2}\left(Y_{n}: \mathbf{Q}\right)$ is an epimorphism.

Since $t-1$ is an isomorphism on $T\left(X_{\infty}^{i}\right)$, the sequence

$$
0 \rightarrow F\left(X_{\infty}^{i}\right) \stackrel{t-1}{\longrightarrow} F\left(X_{\infty}^{i}\right) \rightarrow H_{2}\left(X^{i}\right) \rightarrow 0
$$

shows that $\operatorname{rank}_{\Gamma} F\left(X_{\infty}^{i}\right)=2 g$ where $g$ is the genus of $J_{i}$. The same is true for $Y_{\infty}$ and the similar exact sequences for $t^{n}-1$ show that $\operatorname{dim}_{\mathbf{Q}} H_{2}\left(X_{n}^{i}\right)=$ $2 g n=\operatorname{dim}_{\mathbf{Q}} H_{2}\left(Y_{n}\right)$. Since $i_{*}: H_{2}\left(X_{n}^{i}\right) \rightarrow H_{2}\left(Y_{n}\right)$ is an epimorphism of vector spaces of the same dimension, it is an isomorphism.

A Mayer-Vietoris sequence shows that $i_{*}: H_{2}\left(M_{i} ; \mathbf{Q}\right) \rightarrow H_{2}(W: \mathbf{Q})$ is an isomorphism. Also we have the following Mayer-Vietoris sequences with $\mathbf{Q}$ coefficients:

$$
\begin{aligned}
& \begin{array}{ccc}
H_{2}\left(M_{i}\right) & \rightarrow & H_{1}\left(\partial N\left(k_{j}^{i}\right)\right) \\
\downarrow \cong & & \downarrow \cong
\end{array} \\
& H_{2}(W) \quad \rightarrow \quad H_{1}\left(\partial N\left(E_{j}\right)\right) \\
& \rightarrow H_{1}\left(N\left(k_{j}^{i}\right)\right) \oplus H_{1}\left(M_{i} \backslash \operatorname{Int} N\left(k_{j}^{i}\right)\right) \rightarrow 0 \\
& \cong \downarrow \\
& \rightarrow H_{1}\left(N\left(E_{j}\right)\right) \oplus H_{1}\left(W \backslash \operatorname{Int} N\left(E_{j}\right)\right) \quad \rightarrow \quad 0
\end{aligned}
$$

It follows that

$$
i_{*}: H_{1}\left(M_{i} \backslash k_{j}^{i}: \mathbf{Q}\right) \rightarrow H_{1}\left(W \backslash E_{j}: \mathbf{Q}\right)
$$

is an isomorphism for $i=0,1$ and the lemma follows. Q.E.D.

Proof of Theorem 3. We use the same notation as in the proof of Lemma 1. Consider the following diagram with $\mathbf{Q}$-coefficients:

$$
\begin{aligned}
& H_{1}\left(k_{j}^{0}\right) \rightarrow H_{1}\left(M_{0} \backslash k_{0}^{0}\right) \cong \mathbf{Q} \\
& H_{1}\left(E_{j}\right) \rightarrow H_{1}\left(W \backslash E_{0}\right) \cong \mathbf{Q} \\
& \stackrel{\uparrow}{H_{1}\left(k_{j}^{1}\right)} \rightarrow \stackrel{\uparrow}{\rightarrow} H_{1}\left(M_{1} \backslash k_{0}^{1}\right) \cong \mathbf{Q}
\end{aligned}
$$

Each vertical homomorphism is induced from an inclusion map and an isomorphism in Q-coefficients. Hence the top homomorphism is zero iff so is the bottom homomorphism. Therefore $\xi_{j}^{n}$ is well-defined. Q.E.D.

Example. Let $L_{m}=K_{m, 0} \cup K_{m, 1}$ be an "untwisted spun link" indicated in Figure 1 , where $m$ is a positive integer. (Regard $S^{4}$ as $B^{3} \times S^{1} \cup S^{2} \times B^{2}$. 


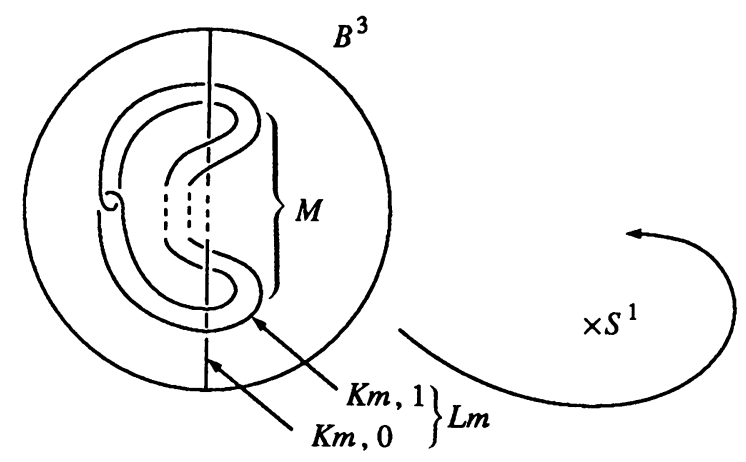

FIGURE 1

The circle in the 3-ball becomes a torus in $S^{4}$ after spinning, and the spun arc together with two disks in $S^{2} \times B^{2}$ forms an $S^{2}$ in $S^{4}$.) . Thus $K_{m, 0}$ is homeomorphic to $S^{2}$, and $K_{m, 1}$ to a torus. Furthermore, $K_{m, 0}$ and $K_{m, 1}$ are unknotted. One can calculate

$$
\xi_{j}^{n}\left(L_{m}\right)= \begin{cases}1 & \text { if } j \equiv \pm m(\bmod n) \\ 0 & \text { otherwise }\end{cases}
$$

for any prime power $n$. Hence $L_{m}$ and $L_{m^{\prime}}$ are not cobordant to each other unless $m=m^{\prime}$. Note that the Sato-Levine invariant vanishes and Cochran's derivative is trivial for any $m$.

\section{ACKNOWLEDGMENT}

The author is grateful to Professor C. McA. Gordon for his encouragement and valuable conversations. The author is also thankful to the referee for pointing out mistakes in an earlier version.

\section{REFERENCES}

[C] T. D. Cochran, Geometric invariants of link cobordism, Comment. Math. Helv. 60 (1985), 291-311.

[S] N. Sato, Cobordisms and semi-boundary links, Topology Appl. 18 (1984), 225-234.

[S-S] Y. Shinohara and D. W. Sumners, Homology invariants of cyclic coverings with applications to links, Trans. Amer. Math. Soc. 163 (1971), 101-121.

[Sum] D. W. Sumners, On the homology of finite cyclic coverings of higher dimensional links, Proc. Amer. Math. Soc. 46 (1974), 143-149.

Department of Mathematics, The University of Texas at Austin, Austin, TeXas 78712 